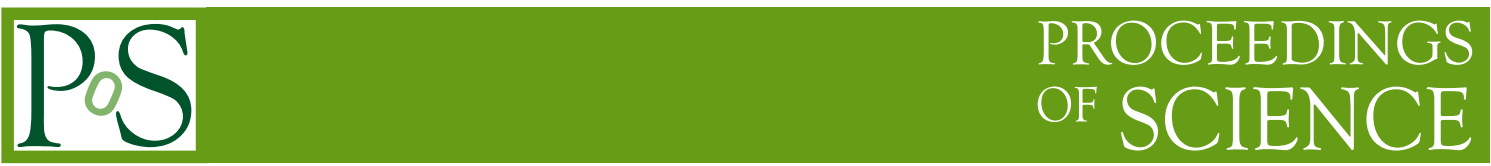

\title{
Signatures of superfluid neutron-star dynamics
}

\section{Brynmor Haskell ${ }^{* i}$}

University of Amsterdam

E-mail: B.D.L.Haskell@uva.nl

\begin{abstract}
In this paper I will discuss how X-ray observations can be used to constrain the gravitational wave emission mechanisms and interior dynamics of superfluid neutron stars in accreting systems. I will briefly review the multifluid formalism for modelling superfluid neutron stars and then focus in particular on the r-mode instability. I will show that the "minimal" scenario, i.e. a neutron star composed exclusively of neutrons, protons (non superconducting) and electrons is not consistent with the observed temperatures and spins of the Low Mass X-ray Binaries, unless the crust is significantly more rigid than expected. An alternative is that the neutron star core may be contain hyperons or that it may be in a type II superconducting state, giving rise to strong vortex/flux tube interactions. Finally I will discuss how future X-ray missions such as LOFT or IXO and gravitational wave observations could help distinguish between the models.
\end{abstract}

Fast $X$-ray timing and spectroscopy at extreme count rates

February 7-11, 2011

Champéry, Switzerland

* Speaker.

$\dagger$ I acknowledge support from the European Union via a Marie Curie fellowship. 


\section{Introduction}

Neutron stars (NSs) represent one of the best laboratories to test our understanding of matter at high densities. With cores that exceed the nuclear saturation density they can allow us to probe the low temperature, high density sector of the QCD phase diagram, which cannot be studied in particle accelerators and heavy ion colliders. Furthermore, at the extreme densities that characterise NS interiors, matter is believed to be in a superfluid/superconducting state once the star has cooled below $\approx 10^{9} \mathrm{~K}$, which will happen very shortly after its birth. This clearly affects the dynamics of the system, as superfluid neutrons will rotate by forming a quantised array of vortices, while protons are likely to form a type II superconductor, thus carrying the stellar magnetic field in quantised flux tubes[1]. In fact NS superfluidity is thought not only to be at the heart of several phenomena, such as radio pulsar glitches, but also to play a significant role in several gravitational wave $(\mathrm{GW})$ emission mechanisms and in determining the response of the star to an external torque in accreting X-ray binaries.

Naturally such rich and complex physics does not come without its challenges. Modelling a superfluid/superconducting NS requires us to follow several massive components (neutrons, protons, possibly hyperons or deconfined quarks) weakly coupled by superfluid and dissipative effects. Although the Newtonian framework for such a task has been developed (see e.g. [2]), a General Relativistic formulation, including the effects of dissipation and vortex dynamics, is still not complete (for a review see [3]). Furthermore several microphysical inputs are required for the models, such as the equation of state, transport coefficients, superfluid gaps etc., all of which are highly uncertain at supranuclear densities.

Clearly a multi-disciplinary effort is required to tackle this problem, with contributions coming from the nuclear physics, neutron star modelling and astrophysical communities. In this paper I will show how inputs from X-ray observations can be used, together with (future) GW observations, to constrain the physics of NS interiors. In particular I will discuss the GW emission driven r-mode instability.

\section{The multifluid model}

Let us first of all present the Newtonian multifluid formalism that will be used to discuss superfluid NSs. Following [2] we can write the equations of motion as coupled Euler equations and continuity equations for each species $\mathrm{x}$ (where $\mathrm{x}$ can indicate neutrons, protons etc...) :

$$
\begin{aligned}
& \partial_{t} n_{\mathrm{x}}+\nabla_{i}\left(n_{\mathrm{x}} v_{\mathrm{x}}^{i}\right)=\Gamma_{\mathrm{x}} \\
& f_{i}^{\mathrm{x}}=\partial_{t} \pi_{i}^{\mathrm{x}}+\nabla_{j}\left(v_{\mathrm{x}}^{j} \pi_{i}^{\mathrm{x}}+D_{i}^{\mathrm{x} j}\right)+n_{\mathrm{x}} \nabla_{i}\left(\mu_{\mathrm{x}}-\frac{1}{2} m_{\mathrm{x}} v_{\mathrm{x}}^{2}+m_{\mathrm{x}} \Phi\right)+\pi_{j}^{\mathrm{x}} \nabla_{i} v_{\mathrm{x}}^{j} .
\end{aligned}
$$

The momentum of each species (or "fluid") is:

$$
\pi_{i}^{\mathrm{x}}=g_{i j}\left[m_{\mathrm{x}} n_{\mathrm{x}} v_{\mathrm{x}}^{j}-2\left(\sum_{\mathrm{y}} \alpha^{\mathrm{xy}} w_{\mathrm{xy}}^{j}\right)\right],
$$

where $n_{\mathrm{X}}$ is the number density of the species, $\mu_{\mathrm{x}}$ its chemical potential, $m_{\mathrm{X}}$ its mass and $v_{\mathrm{X}}^{i}$ its velocity and the parameter $\alpha^{\mathrm{xy}}$ encodes the so-called "entrainment" effect, which leads to a nondissipative coupling between the different components. I use covariant notation, and $g_{i j}$ is the 

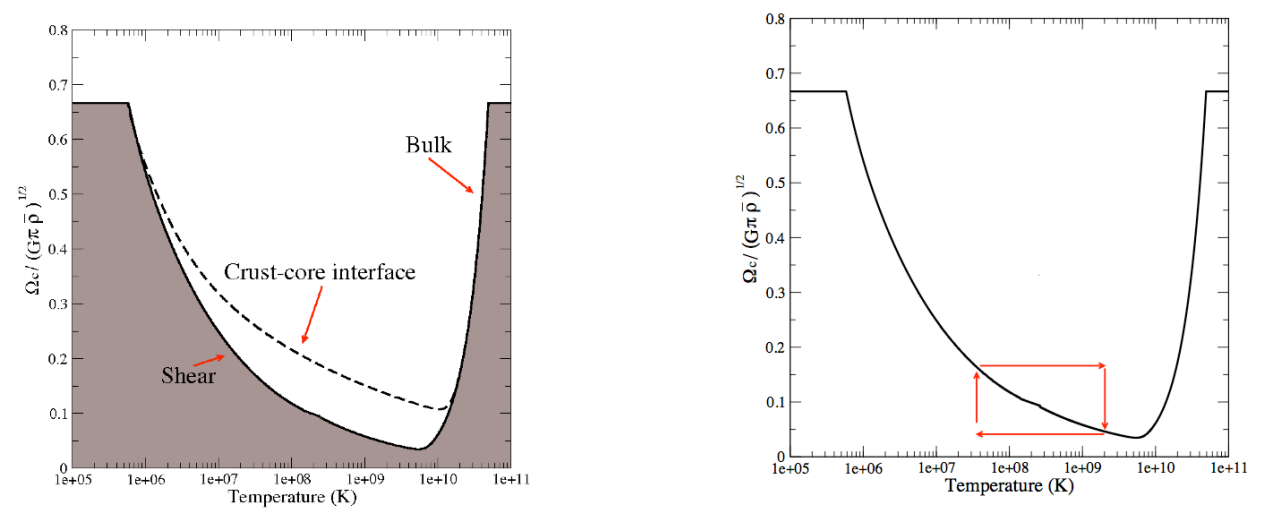

Figure 1: Left panel: The r-mode instability window for a $1.4 M_{\odot}$ NS with a $10 \mathrm{~km}$ radius, assuming an $n=1$ polytrope for the equation of state and a neutron, proton and electron core. Right panel: A typical thermal instability cycle. The star spins up into the unstable region, after which it will heat up rapidly from the shear due to the mode until the thermal runaway is halted by neutrino emission. At this point it spins down below the instability curve, and the cycle starts again.

three-dimensional flat-space metric. The dissipative effects, such as bulk and shear viscosity, are encoded in the tensor $D_{i}^{\mathrm{x}, j}$, while $f_{i}^{\mathrm{x}}$ reprensents the sum of all external forces, in particular the superfluid mutual friction force $([2,4,5])$. The gravitational potential $\Phi$ obeys the Poisson equation $\Delta \Phi=4 \pi G \sum_{\mathrm{x}} \rho_{\mathrm{x}}$, and the system is closed by supplying an equation of state.

We shall mainly be interested in the r-mode instability. An r-mode is a toroidal mode of oscillation of the NS, for which the restoring force is the Coriolis force. It is particularly interesting in this context as it has been shown to be generically unstable to GW emission ([6, 7]) and has been suggested to play a role in the spin evolution of Low Mass X-ray Binaries (LMXBs). In the following sections the analysis of the r-mode instability will be carried out by integrating the linearised version of the equations in (2.1) and (2.2) and then estimating the damping timescale due to various mechanisms, such as hyperon bulk viscosity or mutual friction, following an integral approach, as outlined in [8].

\section{Gravitational waves from LMXBs and the r-mode instability}

LMXBs were first suggested as sources of GWs more than thirty years ago $([9,10])$ and the idea was revived by Bildsten [11] to explain the apparent cutoff in the spin-distribution of these systems (and of the millisecond radio pulsars) at around $700 \mathrm{~Hz}([12,13])$, which is well below the Keplerian breakup limit. The main emission mechanism that could be at work in these systems are essentially two: either a quadrupolar "mountain" is present, sustained by the crust ([11, 14]), the core $([15,16])$ or the magnetic field $([17,18])$ or one could have a mode (and the r-mode is the best candidate) being driven unstable by GW emission ([19]).

Let us concentrate on the latter mechanism and examine the conditions under which the instability can occur. The main constraint is that timescale on which GWs drive the mode, $\tau_{G W}$ must be shorter than the timescale on which viscosity acts to damp it, $\tau_{V}$. Usually this is illustrated in terms of the critical curve on which $\tau_{G W}=\tau_{V}$. The damping timescale $\tau_{V}$ is set by the fastest process at 
a given spin rate and temperature and is such that $\frac{1}{\tau_{V}}=\sum_{i} \frac{1}{\tau_{i}}$, where the subscript " $i$ " labels the various dissipative processes that are at work, i.e. shear viscosity, bulk viscosity, mutual friction etc.. In figure (1) I show the typical instability window for a "minimal" neutron star model. I assume a core of neutrons, non-superconducting protons and electrons and the damping at high temperature is taken to be due to bulk viscosity from modified URCA reactions while at low temperature it is mainly due to shear at the crust/core interface [20]. For such a model the r-mode will however undergo a thermal instability [21]. The star will heat up rapidly from the shear due to the mode until the thermal runaway is halted by neutrino emission, after which the star rapidly spins down below the instability curve, as shown in figure (1). The duty cycle for such a process is very small, with the system spending less than a few percent of the time, depending on the saturation amplitude of the mode, in the unstable region where it is emitting GWs. Let us thus examine how observational estimates of NS spins and temperatures compare to this picture.

\section{Neutron star core temperatures}

It is clear from the discussion in the preceding section that in order to understand a system's behaviour with respect to the r-mode instability we need to estimate the NS's core temperature. This is not a straightforward task, as the stellar interior cannot be probed directly, but rather we need to map the observed surface emission to a core temperature. To do this we shall assume that the core and crust of the star are nearly isothermal (which is very nearly the case if the conductivity of the crust is high, as indicated by recent cooling observations of X-ray transients [22]) and thus assume that the core temperature is simply that at the base of the 'heat blanketing' envelope. As we are considering accreting systems we shall use the relation between the effective surface temperature and interior temperature given in [23]:

$$
\left(\frac{T_{\text {eff }}}{10^{6} \mathrm{~K}}\right)^{4}=\left(\frac{g}{10^{14} \mathrm{~cm} \mathrm{~s}^{-2}}\right)\left(18.1 \frac{T}{10^{9} \mathrm{~K}}\right)^{2.42}
$$

Where $T_{\text {eff }}$ is the observed temperature, obtained from spectral fitting, and $g$ the gravitational acceleration at the NS surface. We can then use X-ray observations of LMXBs in quiescence (when most of the thermal emission is thought to come directly from the NS surface rather than from the disc) to obtain an estimate of the core temperature by assuming a mass and radius for the star. In particular we shall take $M=1.4 M_{\odot}$ and a $R=10 \mathrm{Km}$.

In figure (2) I show the inferred temperatures and spins of the LMXBs for which we have estimates of both (from [24, 25, 26, 13]), with respect to the critical curve for the "minimal" NS model. It is clear that, even with the inherent uncertainties in the temperatures, several systems appear to be well inside the instability window. Given the short duty cycle we do not expect to 'catch' many systems in the unstable region and, furthermore, one of the systems, IGR 00291, has a measured spin up in outburst and spin down in quiescence, which is consistent with a magnetic field of approximately $10^{8} \mathrm{G}$ and shows no indication of additional $\mathrm{GW}$ torques.

This clearly indicates the need for additional physics in our model (as do theoretical calculations of NS temperatures in the presence of an unstable r-mode [27]). The right panel of (2) shows what the effect of hyperon bulk viscosity [28], strong mutual friction [4] (such as that due to the vortex/flux tube interaction in the core) or a rigid crust [29] would be on the instability window. 

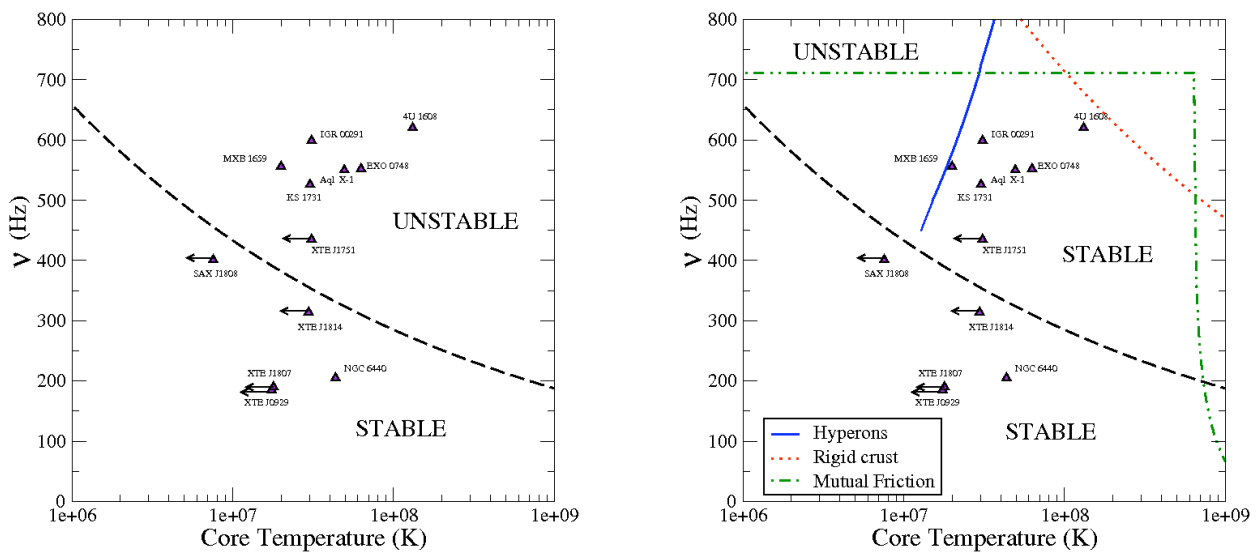

Figure 2: The left panel shows the estimated core temperatures of the LMXBs with respect to the "minimal" instability curve. Clearly many systems would be in the unstable region, which is unexpected. The right panel shows the effect of different physical mechanisms which could be at work to stabilise the r-mode. In particular I show the effect of a rigid crust, with the slippage factor of [29] taken to be 0.5 , of hyperon bulk viscosity from [28] with the parameter $\xi=1$ and of strong mutual friction from [4] with $\mathscr{R}=0.01$ for weak superfluidity.

All these additional mechanism could make the observed systems stable and it is thus imperative to obtain further observational constraints to distinguish between the models. Furthermore if, for example, the instability curve rises with temperature between $10^{7} \mathrm{~K}$ and $10^{8} \mathrm{~K}$, the thermal runaway could be halted; resulting in the star reaching an equilibrium along the curve and emitting GWs at a rate that balances the spinup accretion torque [30,31]. Such a scenario would have clear observational consequences on the timing as there would be an additional spin down torque. There have been suggestions that the spin of SAX J1808 may be constant during outburst [32], even though recent assessments of the spin evolution of this system, together with XTE 1814, do not suggest that there is a need for extra GW spin down torques to explain this behaviour [33].

\section{Conclusions}

We have shown how current X-ray observations can be used to constrain the physics of the r-mode instability window, and thus of the complex physics of NS interiors. In order to truly constrain the models it is however necessary to obtain further observations of NS temperatures and spins. Timing of these systems is also crucial, a task for which the proposed X-ray missions LOFT and IXO would be ideally suited. This is especially important as the GW signature of such events is likely to be very challenging to detect, and detailed modelling is thus crucial to extract the signal from the background noise of the detectors [34].

\section{References}

[1] G. Baym, C. J. Pethick, D. Pines, Nature (London) 224, 673 (1969)

[2] N. Andersson, G. L. Comer, CQG 23, 5505 (2006) 
[3] N. Andersson, G. L. Comer, "Relativistic Fluid Dynamics: Physics for Many Different Scales", Living Rev. Relativity 10, (2007), 1. URL (cited on 15/04/2011): http://www.livingreviews.org/lrr-2007-1

[4] B. Haskell, N. Andersson, A. Passamonti, MNRAS 397, 1464 (2009)

[5] T. Sidery, N. Andersson, G. L. Comer, MNRAS 368, 162 (2006)

[6] N .Andersson, Ap.J.502, 708 (1998)

[7] J. L. Friedman, S. M. Morsink, Ap.J.502, 714 (1998)

[8] N. Andersson, K. Glampedakis, B. Haskell, Phys.Rev.D 79, 103009 (2009)

[9] J. Papaloizou, J. E. Pringle, MNRAS 184, 501 (1978)

[10] R. V. Wagoner, 1984, Ap.J. 278, 345 (1984)

[11] L. Bildsten, Ap.J. 501, L89 (1998)

[12] D. Chakrabarty, E. H. Morgan, M. P. Muno, D. K. Galloway, E. Wijnands, M. van der Klis, C. B. Markwardt, Nature 424, 42 (2003)

[13] A. Patruno, Ap.J. 722, 909 (2010)

[14] G. Ushomirsky, C. Cutler, L. Bildsten, MNRAS 319, 902 (2000)

[15] B. J. Owen, Phys,Rev,Lett. 95, 211101 (2005)

[16] B. Haskell, N. Andersson, D. I. Jones, L. Samuelsson, Phys.Rev.Lett. 99, 231101 (2007)

[17] C. Cutler, Phys.Rev.D 66, 084025 (2002)

[18] A. Melatos, D. J. Payne, Ap.J 623, 1044 (2005)

[19] N. Andersson, K. D. Kokkotas, N. Stergioulas, Ap.J. 516, 307 (1999)

[20] N. Andersson, K. D. Kokkotas, V. Ferrari, Int.Journ.Mod.Phys.D 10, 381 (2001)

[21] Y. Levin. Ap.J. 517, 328 (1999)

[22] E. F. Brown, A. Cumming, Ap.J. 698, 1020 (2009)

[23] A. Y. Potekhin, G. Chabrier, D. G. Yakovlev, A\&A 323, 415 (1996)

[24] C. O. Heinke, P. G. Jonker, R. Wijnands, R. E. Taam, Ap.J. 660, 1424 (2007)

[25] C. O. Heinke, P. G. Jonker, R. Wijnands, C. J. Deloye, R. E. Taam, Ap.J. 692, 584 (2009)

[26] N. Degenaar, M. T. Wolff, P. S. Ray, K. S. Wood, J. Homan, W. H. G. Lewin, P. G. Jonker, E. M. Cackett, J. M. Miller, E. F. Brown, R. Wijnands, MNRAS 412, 1409 (2011)

[27] W. C. G. Ho, N. Andersson, B. Haskell, Phys.Rev.Lett. 107, 101101 (2011)

[28] B. Haskell, N. Andersson, MNRAS 408, 1897 (2010)

[29] K. Glampedakis, N. Andersson, Phys.Rev.D 74, 044040 (2006)

[30] N. Andersson, D. I. Jones, K. D. Kokkotas, MNRAS 337, 1224 (2002)

[31] M. Nayyar, B. J. Owen, Phys.Rev.D 73, 84001 (2006)

[32] J. M. Hartman, A. Patruno, D. Chakrabarty, C. B. Markwardt, E. H. Morgan, M. van der Klis, R. Wijnands, Ap.J. 702, 2673 (2009)

[33] B. Haskell, A. Patruno, Ap.J. 738, L14 (2011)

[34] A. L. Watts, K. Badri, L. Bildsten, B. F. Schutz, MNRAS 389, 839 (2008) 